\title{
INHIBIDORES DE ENZIMA CONVERTIDORA DE ANGIOTENSINA DISMINUYENDO RIESGO DE INFARTO AGUDO DE MIOCARDIO EN DIABÉTICOS.
}

\section{INHIBITORS OF ANGIOTENSIN-CONVERTING ENZYME DECREASING RISK OF ACUTE MYOCARDIAL INFARCTION IN DIABETICS.}

\author{
*Oscar Leonel Bonilla Medina, *Gabriel Emilio Morales Chávez, *Marely Elizabeth Oviedo Morales, \\ *Gilda Gabriela Bonilla Hernández, *Elisa Alejandra Rodríguez Moreno, \\ **Lourdes Azucena Andrade Mancias.
}

\section{RESUMEN}

La enfermedad coronaria ateroesclerótica es el compromiso patológico más frecuente del corazón de los pacientes que sufren Diabetes Mellitus; dicha complicación ha conllevado a la necesaria estimación del riesgo cardiovascular global en pacientes con dicha patología para poder realizar una mejor intervención terapéutica y así disminuir la incidencia de las complicaciones microvasculares y macrovasculares que engloba la variable seleccionada, el infarto agudo de miocardio. En la intervención terapéutica se incluye el tratamiento con Inhibidores de la enzima convertidora de angiotensina que, según estudios, se debe considerar ante todo en pacientes con infarto agudo de miocardio y con diabetes mellitus, o sea, que la implementación de esta familia farmacológica es de elección y constituyen el tratamiento de primera línea en la prevención secundaria para estos pacientes mencionados. Se debe valorar el nivel de evidencia científica existente, sobre la acción de los inhibidores de la enzima convertidora de angiotensina en la disminución del riesgo a padecer un suceso cardiovascular ya que a menudo este cuadro agudo se produce repentinamente y conducen a la

*Estudiantes de sexto año de la carrera de medicina de la Escuela Universitaria de las Ciencias de la Salud de la Universidad Nacional Autónoma de Honduras en el Valle de Sula (EUCS/UNAH-VS)

Dirigir correspondencia a: leonel963008@gmail.com

https://orcid.org/0000-0002-6020-0472

mrodriguezelisa@gmail.com https://orcid.org/0000-0002-3600-3129 gemch27@gmail.com https://orcid.org/0000-0003-1505-9809 gbonilla512@gmail.com https://orcid.org/0000-0002-4700-0580 marely_om@hotmail.com https://orcid.org/0000-0002-8155-0543 **Especialista en Anestesiología / Docente de la EUCS/UNAH-VS. azu3198@yahoo.com https://orcid.org/0000-0002-0271-5081 Recibido: 17 de noviembre 2017 Aprobado: 26 de diciembre del 2018. muerte antes de brindarle la atención requerida. Se realizó revisión exhaustiva de literatura y se citaron las referencias mas relevantes que permitió realizar una revisión bibliográfica descriptiva sobre los inhibidores de la enzima convertidora de angiotensina.

\section{PALABRAS CLAVE}

Inhibidores de la Enzima Convertidora de Angiotensina, Diabetes Mellitus, Factores de Riesgo, Infarto de Miocardio.

\section{ABSTRACT}

Atherosclerotic coronary disease is the most common pathological compromise of the heart of patients suffering from diabetes mellitus; This complication has led to the necessary estimation of global cardiovascular risk in patients with diabetes mellitus to be able to perform a better therapeutic intervention and thus reduce the incidence of microvascular and macrovascular complications that includes the selected variable, acute myocardial infarction. The therapeutic intervention includes treatment with angiotensin-converting enzyme inhibitors that, according to studies, should be considered first of all in patients with acute myocardial infarction and with diabetes mellitus, thus the implementation of this pharmacological family is a choice and constitute the first-line treatment in secondary prevention for the mentioned parients. The level of existing scientific evidence on the action of angiotensin-converting enzyme inhibitors to reduce the risk of suffering a cardiovascular event should be assessed, since this acute condition often occurs and leads to death before 
to provide the required attention. An exhaustive review of the literature was carried out and the most relevant references were cited, to allowed for a descriptive bibliographic review of the angiotensin-converting enzyme inhibitors.

\section{KEY WORDS}

Angiotensin Converting Enzyme Inhibitors, Diabetes Mellitus, Risk Factors, Myocardial Infarction.

\section{INTRODUCCIÓN}

La Diabetes Mellitus (DM) es un grupo de enfermedades metabólicas caracterizadas por hiperglucemia resultante de defectos en la secreción de insulina, la acción de la insulina, o ambos. ${ }^{(1)}$ Esta es una enfermedad crónica y con una alta prevalencia en el mundo y en nuestro país, lo cual es preocupante porque existe el riesgo latente a corto plazo de que una cantidad importante de pacientes con diabetes mellitus sufra algún evento o enfermedad cardiovascular.(2) La DM tiene alta relación con el desarrollo de la aterosclerosis debido a que la afectación de la pared interior de las arterias provoca un estrechamiento y una disminución de la perfusión sanguínea. ${ }^{(3)}$

En Honduras se estimó una incidencia de enfermedades cardiovasculares de 5.7 por 1000 habitantes en el año del 2001.(4) Dentro de este conjunto de patologías cardiovasculares otro estudio atañe a la DM junto con la obesidad el $33 \%$ de la incidencia de estas en Honduras. ${ }^{(3)}$ Las personas con diabetes corren el mismo riesgo de tener un infarto de miocardio que las personas sin diabetes que ya han tenido uno.(3) Esto se debe a que la obesidad y la insulinorresistencia, exacerban los efectos de la disfunción endotelial, y actúan conjuntamente en la pared arterial para desarrollar la aterosclerosis..(5) por lo tanto, con el objeto de restar la mayor cantidad de factores que lleven a las personas con diabetes mellitus a padecer alguna enfermedad cardiovascular, se utilizan los
Inhibidores de la enzima convertidora de angiotensina(IECA) con las cuales nos referiremos a ellos a lo largo del artículo.

Los IECA son una familia de fármacos que previenen la proliferación mioíntima que ocurre en respuesta a la lesión del endotelio vascular $^{(6)}$ y se utiliza en el manejo no solo de hipertensión arterial (HTA), sino que también de diabetes con el fin prevenir distintas complicaciones cardiovasculares como el infarto agudo de miocardio (IAM).

Esta revisión se hizo con el propósito de proporcionar información a todo el gremio médico acerca de los beneficios que ofrecen los IECAS en una población tan grande como son los pacientes diabéticos.

Para la redacción y edición de esta revisión, primero se definió un bosquejo de lo que incluiría el cuerpo del documento y en base a eso se procedió a la búsqueda de información en distintos buscadores electrónicos como ser: Google Académico, Jurn, Scielo, PubMed, Elsevier, base de datos Med-line. También se consultó a otros sitios electrónicos como Organización Mundial de la Salud (WHO); sitios de revistas como: The New England Journal of Medicine (NEJM), British Medical Journal (BMJ), Journal American Medical Association (JAMA). Entre libros, artículos originales, revisiones bibliográficas y proyectos de investigación se encontraron 63 fuentes bibliográficas, de las cuales se seleccionó 42 , basándonos en su actualidad, calidad y relevancia para nuestra revisión.

\section{DESARROLLO \\ Diabetes}

La diabetes es un grupo de enfermedades metabólicas caracterizadas por hiperglucemia resultante de defectos en la secreción de insulina, en la acción de la insulina, o ambos. ${ }^{(1)}$ De acuerdo con la Organización Mundial de la Salud (OMS), el número de 
personas con diabetes ha aumentado de 108 millones en 1980 a 422 millones en 2014. ${ }^{(7)}$

La diabetes conlleva a una serie de complicaciones microvasculares y macrovasculares. Dentro de las primeras tenemos la retinopatía, nefropatía y neuropatía diabética, habitualmente son asintomáticas; y las complicaciones macrovasculares que incluyen las manifestaciones clínicas de la aterotrombosis (macroangiopatía de las arterias de mediano y gran calibre), es decir, la enfermedad cardiaca coronaria (ECC), la enfermedad cerebrovascular y la arteriopatía periférica. El $80 \%$ de la mortalidad es atribuible a las manifestaciones macrovasculares de la enfermedad, precisamente la ECC. ${ }^{(8)}$

\section{Infarto Agudo de Miocardio}

EI IAM es un evento de necrosis miocárdica causado mas frecuentemente por ruptura de una placa ateromatosa, provocando así una isquemia grave como consecuencia de la disminución de flujo coronario. Si bien es cierto que, en su mayoría, el IAM es causado por la ruptura de placa aterosclerótica, existen otros desencadenantes de IAM.

Este puede tener diferentes etiologías que se clasifican de la siguiente manera: $(9,10)$

1. Debido a una falta en el balance de oferta y demanda de oxígeno miocárdico, como en las arritmias, anemias, espasmos, etc.

2. Infarto relacionado con una intervención coronaria percutánea (ICP).

3. Infarto relacionado con procedimientos quirúrgicos de revascularización coronaria.

Las lesiones formadas en la aterosclerosis tienen 3 fases: estría grasa (compuesta principalmente por las células espumosas), placa ateromatosa fibrosa y lesión complicada. Las últimas dos son las causantes de las manifestaciones clínicas de la enfermedad. ${ }^{(11,12)}$
Las estrías grasas son zonas delgadas, planas y de coloración amarilla en la íntima que crecen de manera progresiva para convertirse en lesiones más gruesas y un poco elevadas mientras crecen en longitud. En el examen histológico consisten en macrófagos, células musculares lisas distendidas con lípidos para formar células espumosas.

Si no hay resolución del daño local, se disparan los procesos de inmunidad adaptativa y se reclutan células $\mathrm{T}$ y $\mathrm{B}$ a la zona. Por su parte, las células musculares lisas vasculares en la capa media de las arterias se diferencian e inician su migración hacia la íntima donde, además de producirse cambios en su expresión de genes, cambian la regulación de la vasotonicidad de la zona lesionada. ${ }^{(11,12)}$

Una vez el endotelio se daña, los monocitos circulantes y lípidos empiezan a adherirse al área. Esta placa ateromatosa fibrosa se caracteriza por un color gris blanco perlado producido por los macrófagos que ingieren y oxidan las lipoproteínas acumuladas y forman una estría grasa visible. Con el tiempo las estrías grasas crecen y proliferan al músculo liso. Conforme aumenta el tamaño de las lesiones, invaden el lumen de la arteria. Los macrófagos liberan sustancias que causan inflamación y al final pueden ocluir un vaso o predisponer la formación de trombos lo que reduce el flujo sanguíneo. ${ }^{(11,12)}$

Por tanto, la lesión aterosclerótica es el producto de una infiltración de lípidos que da origen a una respuesta inflamatoria especializada a diferentes cambios en la pared vascular. El carácter crónico del proceso de infiltración lipídica, por la presencia de altas concentraciones de lipoproteínas de baja densidad circulantes, conduce a la formación de placas que, en fases avanzadas y con complicaciones trombóticas, pueden ocluir las arterias y producir sintomatología clínica. ${ }^{(11,12)}$ 
Es imperativo mencionar que se conocen otros factores de riesgo asociados al IAM y estos son: tabaco, hipertensión, factores genéticos, hipercolesterolemia y el sedentarismo. ${ }^{(13)}$ y que la diabetes no se considera como el único factor de riesgo de importancia para llegar a padecer un IAM, pero si uno muy contribuyente.

\section{Riesgo Cardiovascular en Diabéticos}

La DM especialmente el tipo 2, es una enfermedad metabólica en la que coexisten diversos factores, convergiendo estos en un riesgo cardiovascular de 2 a 3 veces superior al de la población general masculina y 2 a 6 veces superior en la población general femenina. La principal causa de muerte entre la población diabética es el infarto agudo de miocardio, seguida por el cáncer. ${ }^{(2,14,15)}$ La obesidad y la insulinorresistencia, exacerban los efectos de la disfunción endotelial, y actúan conjuntamente en la pared arterial para desarrollar la aterosclerosis. ${ }^{(5)}$

La enfermedad coronaria (EC) ateroesclerótica es el compromiso patológico más frecuente del corazón en la DM, duplicando o triplicando el de la población general. ${ }^{(16)} \mathrm{El}$ mayor riesgo de IAM lo exhiben los pacientes que padecen de DM con enfermedad

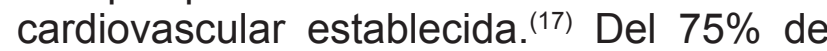
los pacientes diabéticos que fallece por enfermedad cardiovascular, una proporción importante tenia complicaciones, siendo el pronóstico de un evento coronario en el corto y largo plazo el más ominoso. ${ }^{(18)}$

La hipertensión arterial (HTA) es un síndrome de etiología múltiple caracterizado por la elevación persistente de las cifras de presión arterial en cifras iguales o mayores a 140/90 ml/Hg. ${ }^{(19)}$ Esta patología provoca una duplicación del riesgo de enfermedades cardiovasculares como es la cardiopatía coronaria. ${ }^{(9)}$ Estudios realizados en pacientes con diabetes mellitus tipo 2 , se observó que la comorbilidad más frecuente de estos pacientes es la HTA con una frecuencia del $64 \%$ y las cardiopatías con una frecuencia de $1.83 \%$. $^{(20)}$

En las personas con prediabetes, la prevalencia de la HTA es el doble que en personas con tolerancia normal a la glucosa (el 67,9 frente al $31,6 \%$ ) y aún mayor en diabéticos $(79,4 \%){ }^{(21)}$

Al determinar el IAM y la HTA como principales complicaciones, consigna a los IECAS como un elemento clave en el manejo de los pacientes con DM con alto riesgo cardiovascular como en el caso de personas de sexo masculino que no realizan actividades físicas. ${ }^{(2)}$

\section{Fisiopatología del ateroesclerosis en la diabetes}

La aterosclerosis, que afecta a las arterias coronarias, cerebrales y periféricas (extremidad inferior) es la causa predominante de mortalidad, siendo responsable del $70 \%$ a $80 \%$ de todos los fallecimientos de los pacientes con esta enfermedad. ${ }^{(10,22)}$ La DM es un factor de riesgo importante para la arteriopatía coronaria, la enfermedad cerebrovascular y la vasculopatía periférica. La prevalencia de estas complicaciones macrovasculares se incrementa entre 2 y 4 veces en personas con diabetes. ${ }^{(22)}$ Muchas anomalías inducidas por el estado diabético pueden contribuir al ateroesclerosis, entre las cuales se pueden enumerar: $(9,23,24)$

\section{Anomalías lipídicas:}

- Aumento de las lipoproteínas de muy baja densidad (VLDL) y lipoproteínas de baja densidad (LDL) totales.

- Aumento de las LDL densas de pequeño tamaño (aterogénicas).

- Disminución de las lipoproteínas de alta densidad(HDL).

- Aumento de la oxidación y de la glucosilación de las lipoproteínas.

- Disminución de la actividad de la lipoproteinlipasa. 
- Aumento de la agregación y adhesión plaquetarias.

- Disfunción de las células endoteliales e inducción de un estado pro coagulante.

El estado hiperglucémico influye directamente en las anomalías lipídicas ya mencionada, específicamente en la oxidación y glucosilación de las lipoproteínas. Es conocido que un aumento de los niveles de Glucosa mantenido en el tiempo produce la glucosilación no enzimática de proteínas circulantes, definiéndose la glucosilación como la reacción de grupos aminos primarios de aminoácidos, péptidos y proteínas con el grupo carbonilo de los azúcares reductores. ${ }^{(24)}$

A lo largo de esta reacción se pueden distinguir etapas: inicialmente se produce la asociación del azúcar con la proteína y como segundo paso se producen los productos de glucosilación avanzada (PGA). La formación de PGA incluye reacciones de deshidratación, condensación cíclica, entrecruzamientos intermoleculares y oxidación por radicales libres del oxígeno, lo que en el caso de las lipoproteínas afecta aún más por la presencia de ácidos grasos poliinsaturados de fácil oxidación dando lugar a lipoproteínas glucosiladas, oxidadas y glucooxidadas que son especialmente aterogénicas. ${ }^{(24)}$

También estos PGA producen enlaces covalentes de colágeno, provocando acumulación y atrapamiento en el subendotelio de proteínas del plasma tales como albúmina, lipoproteína de baja densidad (LDL) e inmunoglobulina G ( $\lg G)$, causando en parte, el estrechamiento luminal, característica importante en los vasos diabéticos. ${ }^{(24)}$

Cuando falta insulina, se invierten todos los efectos promotores del depósito de la grasa descritos con anterioridad. El más importan- te es que la enzima lipasa sensible a la insulina de las células adiposas experimenta una gran activación. Con ello, se hidrolizan los triglicéridos almacenados y se liberan enormes cantidades de ácidos grasos y de glicerol a la sangre circulante. La consecuencia es que las concentraciones plasmáticas de ácidos grasos libres empiezan a ascender a los pocos minutos. ${ }^{(25)}$

El exceso de ácidos grasos del plasma, junto con la falta de insulina, favorece también la conversión hepática de algunos de los ácidos grasos en fosfolípidos y colesterol, dos de los principales productos del metabolismo lipídico. Estas dos sustancias, junto con el exceso de triglicéridos producido al mismo tiempo en el hígado, se liberan hacia la sangre junto con las lipoproteínas. A veces, las lipoproteínas del plasma se triplican cuando falta insulina, por lo que la concentración total de lípidos plasmáticos alcanza varias unidades porcentuales en lugar de la cifra habitual del $0,6 \%$. $^{(25)}$

Por lo que se podemos puntualizar en la fisiopatología del ateroesclerosis en los pacientes con diabetes es: ${ }^{(24,25)}$

- El déficit de insulina provoca la lipólisis de la grasa almacenada, con liberación de los ácidos grasos libres.

- El déficit de insulina aumenta las concentraciones plasmáticas de colesterol y de fosfolípidos.

- Este incremento de los lípidos, sobre todo del colesterol, acelera el desarrollo de aterosclerosis en los enfermos con diabetes grave.

- Estado hiperglucémico mantenido conlleva a la generación y atrapamiento de lipoproteínas glucosiladas aterogenas.

Es por todas las variantes metabólicas inducidas por la diabetes que esta atribuye al riesgo de formación de ateromas y en su consecuencia una patología isquémica. 


\section{Mecanismo de IECA}

Para disminuir la enfermedad cardiovascular de los pacientes diabéticos, se debe tratar los múltiples factores de riesgo que conllevan a esta afección, como la dislipidemia, la hipertensión arterial y el estado protrombótico. El beneficio de la reducción de la presión arterial en pacientes diabéticos ha quedado demostrado en numerosos ensayos clínicos, donde el control intensivo de la presión arterial se asociaba a una reducción del riesgo de mortalidad relacionada con DM. ${ }^{(26)}$

Los IECA son una familia de fármacos que se usan comúnmente en el tratamiento de pacientes hipertensos. Estos actúan como lo dice explícitamente su nombre inhibiendo la enzima convertidora de angiotensina, esencial para la respuesta del Sistema Renina Angiotensina (RAS) que provoca un aumento de la tensión arterial. ${ }^{(27)}$

Las ventajas de atenuar la respuesta del RAS, es el beneficio que se tiene al contrarrestar los efectos negativos que este provoca, entre los cuales tenemos: ${ }^{(27)}$

- Vasoconstricción que en concentraciones circulantes altas de angiotensina II que pueden disminuir el flujo sanguíneo cerebral y coronario.

- El gen del receptor AT1 (subtipo de receptor al que se une a la angiotensina II predominantemente en tejidos vascular y miocárdico) contiene un polimorfismo (transversión de $\mathrm{A}$ a $\mathrm{C}$ en la posición 1166) asociado a hipertensión, miocardiopatía hipertrófica y vasoconstricción coronaria. Además, el alelo $\mathrm{C}$ establece sinergia con el alelo de deleción de la Enzima Convertidora de Angiotensina (ACE), en lo que toca a un mayor riesgo de arteriopatía coronaria.

Los beneficios de reducir la presión arterial (PA) en personas con DM han sido probados fehacientemente en los estudios Hyper- tension Optimal Treatment (HOT) y United Kingdom Prospective Diabetes Study (UKPDS). ${ }^{(28)}$

Aquí es donde los IECAS obtienen su protagonismo para la prevención en la formación de esta placa ateroesclerótica, ya que aparte de frenar los efectos negativos que llegara a tener el RAS, también los IECA previenen la proliferación mioíntima que ocurre en respuesta a la lesión del endotelio vascular. ${ }^{(4)}$

The Eight Joint National Committee (JNC 8) recomienda que la presión arterial en los diabéticos debe controlarse a niveles menores de 140/90 mmHg. El manejo inicial de la hipertensión según el JNC 8 en aquellos pacientes con diabetes deben incluir ya se un IECA o un bloqueador de los receptores de angiotensina (ARB), o un diurético tiazídico o un bloqueador de los canales de calcio (CCB); cualquiera de ellos como monoterapia, en el caso de que falle la monoterapia se continua el algoritmo propuesto por la JNC 8 hasta alcanzar los valores de tensión arterial deseados. ${ }^{(29)}$

No está en demás recalcar que se considera determinante la vigilancia de desarrollo HTA en los pacientes diabéticos, ya que en ese riesgo de HTA se incrementan las posibilidades de sufrir un IAM. Ya que una de las comorbilidades que acompaña a los pacientes con Diabetes mellitus tipo 2 es la hipertensión arterial. ${ }^{(13)}$

En estudios como United Kingdom Prospective Study-39 (UKPDS) desarrollado por Jenny O'Neill Diabetes Centre, Derbyshire Royal Infirmary, Derby, UK (1999), Hypertension Optimal Treatment (HOT) realizado por Hansson L. et al. (1996), Systolic Hypertension in the Elderly Programme (SHEP) realizado por Probstfield JL, Applegate WB et al. (1989), Hypertension in the Very Elderly Trial (HYVET-Pilot) realizado por Christopher Bulpitt et al (1994) indicó que la reduc- 
ción sistólica aislada o sistólica-diastólica en hipertensión reduce significativamente el riesgo de complicaciones vasculares micro y macro y muerte cardiovascular (CV) o muerte relacionada con la diabetes. Por consiguiente, el tratamiento farmacológico para los pacientes con DM e HTA, debe ser con un régimen que incluye un inhibidor de ACE. ' 30 '

\section{Disminución de incidencia de IAM al usar IECA}

En el artículo "Standards of Medical Care in Diabetes" realizado por The American Diabetes Association (2012), se encontró que un IECA reduce la morbimortalidad cardiovascular. ${ }^{(30)}$

En el estudio "Long-Term Follow-up after Tight Control of Blood Pressure in Type 2 Diabetes" desarrollado por Holman R, Paul $S$ et al. (2008) se obtuvo una reducción del riesgo relativo observado durante el tiempo del Infarto agudo de miocardio (21\%) y el riesgo por muerte de cualquier otra causa $(18 \%)$ también disminuyó durante el período de observación. ${ }^{(31)}$

Por tanto, en un paciente diabético con riesgos cardiovasculares aumentados, Ios IECA y otros medicamentos constituyen la piedra angular para la prevención secundaria. ${ }^{(32)}$

Otro caso donde se demuestra el efecto positivo que tienen los IECAS es El subestudio del HOPE (Heart Outcomes and Prevention Evaluation), The Microalbuminuria, Cardiovascular and Renal Outcomes (MICRO) investigó los beneficios de ramipril(IECA), en sujetos con diabetes y enfermedad coronaria versus placebo. Los resultados mostraron una disminución del riesgo relativo de eventos cardiovasculares en $25 \%$, de $22 \%$ en infarto de miocardio, de $33 \%$ en accidentes cerebro vasculares y $37 \%$ en muerte cardiovascular. ${ }^{(33)}$

Dado que múltiples estudios han demostra- do que el control de los factores de riesgo cardiovascular en los diabéticos previene la aparición de complicaciones en los órganos diana, las sociedades científicas internacionales recomiendan un tratamiento integral en el paciente diabético, que asocie el control del perfil glucémico con la prevención de las complicaciones cardiovasculares mediante el uso de inhibidores del enzima de conversión de la angiotensina (IECA). ${ }^{(34)}$

Los IECA pueden ser efectivos en la prevención y hasta en el tratamiento del aterosclerosis establecida. Además de prevenir la proliferación mioíntima que ocurre en respuesta a la lesión del endotelio vascular se ha observado que los IECA reducen la formación de placas aterosclerosas en la aorta descendente de los conejos con aterosclerosis experimental genética cosa que no logran otros antihipertensivos como los beta bloqueadores adrenérgicos o los antagonistas del calcio. Resultados similares a éste se encontraron en animales sometidos a dietas hipercolesterolémicas. ${ }^{(35)}$

El estudio EUROPA comparó el perindopril (IECA) con un placebo donde se llegó a determinar que el tratamiento con perindopril se asocia con una reducción del riesgo relativo del $20 \%$ en infarto de miocardio. ${ }^{(36)}$

Es necesario comentar que la utilidad de los IECA en el IAM no se limita únicamente a disminuir su incidencia en la población diabética, sino que también se ha observado su impacto beneficioso en la reducción de la mortalidad en la persona tanto con diabetes o sin ella luego de haber sufrido un IAM.

En el estudio Mortality benefit of long-term angiotensin-converting enzyme inhibitors or angiotensin receptor blockers after successful percutaneous coronary intervention in non-ST elevation acute myocardial infarction realizado en Portugal encontró que las personas que sufrieron un IAM, tomando en 
cuenta los riesgos, la tasa de mortalidad fue significantemente más baja en pacientes que tomaban sus IECAs/ARB s con un cociente de riesgo de $0.77 \quad(0.63-0.94$; Reducción de riesgo absoluta de $3.8 \%) .{ }^{(37)}$

Dentro de este beneficio de reducir la mortalidad luego de padecer el IAM, el estudio denominado SMILE IV concluyó que, los pacientes que usaron Zofenopril vrs otros IECAS después del infarto agudo de miocardio tienen un mejor pronóstico sin importar si tienen o no factores de riesgo como la diabetes. . $^{(36,37,38,39)}$

Debido a esto, es necesario tener en cuenta durante el manejo inicial de un paciente que ha sufrido un IAM la indicación de IECA en el control inicial de este paciente. ${ }^{(40,41)}$

\section{Conclusiones}

Con los nuevos estilos de vida que ha acarreado la modernización de la sociedad, en donde todo está pensado para facilitar las actividades cotidianas, el ser humano se ha vuelto sedentario, factor que aumenta la prevalencia de obesidad, diabetes y enfermedades cardiovasculares.
En Honduras la de Diabetes Mellitus se presenta aproximadamente en el $12 \%$ de la población siendo una de las primeras 10 causas de muerte, lamentablemente solo el $50 \%$ de esta población conocen que son diabéticos $\mathrm{y}$, de este $50 \%$ solo la mitad tienen acceso al tratamiento.

La enfermedad coronaria ateroesclerótica es el compromiso patológico más frecuente del corazón en la diabetes mellitus, siendo el infarto agudo miocardio la causa más frecuente de muerte en estos pacientes. El uso de IECAS en pacientes diabéticos tiene el fin de prevenir distintas complicaciones cardiovasculares; en el caso de infarto agudo de miocardio evitando la formación de la placa ateroesclerótica, consecuencia de la alteración metabólica producida por la diabetes y previniendo la proliferación mioíntima que ocurre en respuesta a la lesión del endotelio vascular. Los IECAS se utilizan en el tratamiento de la hipertensión arterial que es una enfermedad muy ligada a la diabetes y un factor de riesgo de infarto agudo de miocardio en los pacientes diabéticos.

Por lo tanto, los IECAS se pueden considerar como tratamiento para limitar los eventos de IAM en pacientes diabéticos.s eventos de IAM en pacientes diabéticos. 


\section{REFERENCIAS BIBLIOGRÁFICAS}

1. American Diabetes Association. Diagnosis and Classification of Diabetes Mellitus. Diabetes Care. 2014; 37(Supplement 1): S81-S90. DOI https:// doi.org/10.2337/dc14-S081.

2. García Barreto D, Toruncha chukram A. Los inhibidores de la enzima conversora de angiotensina. Rev Cubana Cardiol Cir Cardiovas[Internet]. 1997 [Consultado14 Abril 2017];11(1): 29-46.

Disponible en: http://163.178.103.176/ CasosBerne/7gRenal/Caso37-2/HTML/ CasosB2/3/ca3.htm.

3. Feng $A$, Peña Y, Li W. La cardiopatía isquémica en pacientes diabéticos y no diabéticos. Rev Haban Cienc Méd [Internet]. 2017 [consultado 17 mayo 2017]; 16(2): [216-227]. Disponible en: http://www.revhabanera.sld.cu/index. php/rhab/article/view/1781.

4. Ramírez E, Medina $M$, Sierra $M$, Thompson A, Retes E, Martinez L, y Banegas L. Prevalencia y factores de riesgo asociados a enfermedad cerebro vascular en un área rural de Honduras: un estudio poblacional de casos y controles. Rev Méd PostGrad Med UNAH. 2007; 10(1): 10-26.

5. Vicente Sánchez B, Vicente Peña E, Costa Cruz M. Estimación del riesgo cardiovascular en pacientes con diabetes tipo 2: una explicación necesaria. Rev Finlay [Internet]. 2015 [Consultado 18 de mayo 2017]; 5(3): 178-189. Disponible en: http://scielo.sld.cu/scielo. php? script=sci_arttext\&pid=S2221 $-24342015000300005 \&$ Ing $=$ es.

6. Cárcamo Mejía S, Pavón Núñez D, Diaz C, Figueroa R, Cortez Flores A, Arguello Mejía D, et al. Caracterización del accidente cerebrovascular adultos jóvenes atendidos en el Hospital Escuela Universitario, Tegucigalpa, Honduras durante los años 2013-2015. RHCS [Internet]. 2017 [Consultado 14 Abril 2017]; 2(2):123-131.

Disponible en: http://uhsalud.com/index. php/revhis pano/article/view/156.

7. Mathers C, Loncar D. Projections of Global Mortality and Burden of Disease from 2002 to 2030. PLoS Med. 2006; $3(11): \mathrm{e} 442$.

8. Molina Escribano F. Diabetes mellitus tipo 2 como modelo de aterosclerosis acelerada: Factores asociados y morbimortalidad relacionada en una cohorte prospectiva española.[Tesis]. Madrid: Universidad Autónoma de Madrid. 2015.

9. Blengio Pinto J, Fauci A, Hauser S, Jameson J, Kasper D, Longo D, et al. Harrison principios de medicina interna. 19a ed. México D.F. (México): Mc Graw-Hill; 2016.

10. Borrayo-Sanchez G, Rosas-Peralta M, Pérez -Rodríguez G, Ramírez-Arias E, Almeida-Gutiérrez E, Arriaga-Dávila J. Infarto agudo de miocardio con elevación del segmento ST: Código I. Rev Med Inst Mex Seguro Soc [Internet]. 2018 [Consultado 4 Junio de 2018]; 56(1):26-37. Disponible en: http:// www. medigraphic.com/cgi-bin/new/resumen. cgi?IDARTICULO=77625.

11. Grossman S, Porth C. Porth fisiopatología. 9a ed. Barcelona: L'Hospitalet de Llobregat: Wolters Kluwer Health /Lip pincott Williams \& Wilkins; 2014.

12. Badimon L. Fisiopatología de la pared arterial y papel del colesterol en el origen y progresión de la placa de ateroma. Clínica e investigación en aterosclerosis. 2017;29(Supl. 1):4-8. 
13. Rodríguez Domínguez L, Herrera Gómez V, Dorta Morejón E. Algunos factores de riesgo que favorecen la aparición del infarto agudo del miocardio. Rev Cubana Med Gen Integr [Internet]. 1998 [Consultado 17 enero de 2019] ; 14 (3): 243-249. Disponible en: http:// scielo.sld.cu/scielo.php?script=sci_art text\&pid=S0864-21251998000300008\& Ing=es.

14. Nadal J, Gutiérrez P. ¿Es necesario el tratamiento integral de la diabetes mellitus tipo 2 y los factores de riesgo cardiovascular?. Med Clin (Barc). 2013;141 Suppl 2:7-13.

15. Zavala C, Florenzano F. Diabetes y corazón. Rev Méd Clín Las Condes [Internet]. 2015 [Consultado 5 Marzo de 2017];2(26):175-185. Disponible en: http://www.sciencedirect.com/scien ce/article/pii/S0716864015000383.

16. Booth G, Kapral M, Fung K, Tu J. Relation between age and cardiovascular disease in men and women with diabetes compared with non-diabetic people: a population-based retrospective cohort study. Lancet. 2006; 368(9529): 29-36.

17. Ali M, Bullard K, Saddine J, Cowie C, Imperatore G, Greeg E. Achievement of goals in U.S. diabetes care, 1999-2010. $\mathrm{N}$ Engl J Med 2013; 368(17): 1613-1624.

18. Malmberg $\mathrm{K}$, Yusuf $\mathrm{S}$, Gerstein $\mathrm{H}$, Brown J, Zhao F, Hunt D, et al. Impact of diabetes on long-term prognosis in patients with unstable angina and non-Q-wave myocardial infarction: results of the OASIS (Organization to Assess Strategies for Ischemic Syndromes) Registry. Circulation. 2000; 102(9): 1014-1019.

19. Garcia Flores R, Acosta Quiroz CO,
León Ibarra YM, Lagarda Vega BM, Garcia Hernandez C, Sotelo Quiñonez TI. Efectos de una intervenciónmultidisciplinaria en pacientescon hipertensión arterial primaria. Psicología y Salud. 2018;28(1):95-105.

20. Bermúdez J, Aceituno N, Alvarez G, Giacaman-Abudoj L, Silva-Cárcamo H, Salgado AL. Comorbilidades en los Pacientes con Diabetes Mellitus Tipo 2 del Instituto Nacional del Diabético, Abril-Junio 2016, Tegucigalpa, Honduras. [Internet]. Arch Med [Internet]. 2017 [Consultado 14 Abril 2017]; 12(4): 1-5. Disponible en: http://www.archivosde medicina.com/medicinadefamilia/comor bilidades-en-los-pacientes-con-diabe tes-mellitus-tipo-2del-instituto-nacio nal-del-diabeacutetico-abriljunio-2016 tegucigalpa. php?aid=17875.

21. Menéndez E, Delgado E, Fernández-Vega F, Prieto M, Bordiú E, Calle A, et al. Prevalencia, diagnóstico, tratamiento y control de la hipertensión arterial en España. Resultados del estudio Di@bet.es. Rev Esp Cardiol. 2016; 69(6):572-578.

22. Candelaria Brito J, Cruz González T, Rosa Rieumont E, Acosta Cruz C, Alfonso González Y. Estimación del riesgo cardiovascular global en pacientes con diabetes mellitus. MEDISAN [Internet]. 2013 [Consultado 6 de abril de 2018];17(2):316-323. Disponible en: http://scielo.sld.cu/scielo.php?script= sci_arttext\&pid=S1029-3019201300 0200012\&lng=es.

23. Goldman L, Schafer A, (editors) Goldman's cecil medicine. 25th ed. Philadelphia: Elsevier/Saunders; 2012.

24. Aponte L, Ramírez $\mathrm{R}$, Hernández $\mathrm{S}$, Somontes D. Los procesos de glucosilación no enzimática. AMC [Internet]. 
2009[Consultado 17 de enero de 2017];13(6):151-154. Disponible en: http://www.scielo.sld.cu/scielo.php? script=sci_arttext\&pid=S1025-0255 $2009000600020 \&$ Ing $=$ es.

25. Hall J, Guyton A. Tratado de fisiología médica. 13a ed. Amsterdam: Elsevier; 2016.

26. Pereira Despaigne O, Palay Despaigne M, Rodríguez Cascaret A, Eyra Barros R. Diabetes mellitus and the cardiovascular complications. MEDISAN [Internet]. 2015 Mayo [Consultado18 de mayo de 2017]; 19(5):675-683. Disponible en: http://scielo.sld.cu/scielo. php? script=sci_arttext $\&$ pid $=\mathbf{S} 1029$ $-30192015000500013 \&$ Ing $=$ es.

27. Limbird L, Gilman A, Hardman J. Goodman and Gilman's The pharmacological basis of therapeutics. 13a ed. New York: McMillan ; 2017.

28. Milrad S, Luongo Á, López González E, García A, Houssay S, Ruiz Morosini M, et al. Hipertensión arterial y diabetes: guías de recomendaciones para la práctica clínica. 2015. Comité de Hipertensión Arterial y otros Factores de Riesgo Cardiovascular. Sociedad Argentina de Diabetes. Rev Soc Arg Diabetes. 2015; 49(2): 61.

29. Lloyd-Jones D, Morris P, Ballantyne C, Birtcher K, Daly D, DePalma $S$ et al. 2017 Focused Update of the 2016 ACC Expert Consensus Decision Pathway on the Role of Non-Statin Therapies for LDL-Cholesterol Lowering in the Management of Atherosclerotic Cardiovascular Disease Risk. J Am Coll Cardiol. 2017;70(14):1785-1822.

30. American Diabetes Association. Standards of Medical Care in Diabetes-2012. Diabetes care [Internet]. 2012 [Consul tado 22 de Abril de 2017];35(1):29-33. Disponible en: http://care.diabetesjour nals.org/content/35/Supplement_1/ S11.short.

31. Holman R, Paul S, Bethel M, Neil H, Matthews D. Long-Term Follow-up after Tight Control of Blood Pressure in Type 2 Diabetes. . N Engl J Med. 2008; 359(15): 1565-1576.

32. Achiong Alemañy F, Cobas Pérez M, Achiong Estupiñán $F$, Bello Rodríguez $B$, Fernández A, Rodríguez Betancourt Z. Caracterización en la atención de urgencia del infarto agudo de miocardio en la provincia de Matanzas. Rev Med Electrón [Internet]. 2012 [Consultado 14 de abril de 2017];34(5):531-547. Disponible en: http://scielo.sld.cu/scie lo. php? script $=$ sci_arttext $\&$ pid $=\mathrm{S} 168$ 418242012000500003\&lng=es.

33. Iza Stoll A. Tratamiento de la hipertensión arterial primaria. Acta méd. peruana [Internet]. 2006 Mayo [Consultado 6 de abril de 2018] ; 23(2):93-99.

Disponible en: http://www.scielo.org. pe/scielo.php?script=sci_arttext\&pid= S17 28-59172006000200009\&Ing=es.

34. Agudo Villa T, Álvarez-Rodríguez E, Caurel Sastre Z, Martínez A, Merinero Palomares R, Álvarez Rodríguez V, et al. Prevención de las complicaciones cardiovasculares asociadas a la diabetes mellitus en los servicios de urgencias. Servicio de Urgencias, Hospital Universitario de Getafe, Madrid, España [Internet]. 2015 [Consultado 22 de abril de 2017];(27):151-154. Disponible en: https://www.researchgate.net/profile/ Raul_Merinero/publication/282233 469_Prevention_of_cardiovascular complications_associated_with_diabe tes_mellitus_Hospital_emergency_de partment_involvement/links/56 125 8a608ae0fc513f33153.pdf. 
35. García Barreto D, Toruncha Chuckram A. Los inhibidores de la enzima conversora de angiotensina. Revista Cubana Cardiología y Cirugía Cardiovascular [Internet]. 1997 [Consultado 8 de Mayo de 2018];11:29-46. Disponible en: http://bvs.sld.cu/revistas/car/vol11 _1_97/car04197.htm.

36. Redon J, Mancia G, Sleight P, Schumacher H, Gao P, Pogue J, et al. Safety and efficacy of low blood pressures among patients with diabetes: Subgroup analyses from the ONTARGET (ONgoing Telmisartan Alone and in combination with Ramipril Global Endpoint Trial). J Am Coll Cardiol. 2012; 59(1): 74-83.

37. González Cambeiro M, López López A, Abu Assi E, Raposeiras Roubín S, Peña Gil C, García Acuña J and González Juanatey R. Mortality benefit of long-term angiotensin-converting enzyme inhibitors or angiotensin receptor blockers after successful percutaneous coronary intervention in non-ST elevation acute myocardial infarction. Rev Port Cardiol. 2016; 35(12): 645653.

38. Borghi C, Omboni S, Reggiardo G, Bacchelli S, Degli Esposti D, Ambrosioni E. Efficacy of Zofenopril Compared With Placebo and Other Angiotensin-converting Enzyme Inhibitors in Patients With Acute Myocardial Infarction and Previous Cardiovascular Risk Factors: A
Pooled Individual Data Analysis of 4 Randomized, Double-blind, Controlled, Prospective Studies. J Cardiovasc Pharmacol . 2017;69(1):48-54.

39. Borghi C, Ambrosioni E, Novo S, Vinereanu D, Ambrosio G. Comparison Between Zofenopril and Ramipril in Combination With Acetylsalicylic Acid in Patients With Left Ventricular Systolic Dysfunction After Acute Myocardial Infarction: Results of a Randomized, Double-Blind, Parallel-Group, Multicenter, European Study (SMILE 4). Clin Cardiol [Internet]. 2012 [Consultado 11 de Junio de 2017];35(7):416-423.

Disponible en: http://onlinelibrary.wiley. com/doi/10.1002/clc.22017/full.

40. Coll Muñoz Y, Valladares Carvajal F, González Rodríguez C. Infarto agudo de miocardio. Actualización de la Guía de Práctica Clínica. Rev. Finlay [Internet]. 2016 [Consultado 10 de junio de 2017]; 6(2):170-190.

Disponible en: http://scielo.sld.cu/scielo. php?script=sci_arttext\&pid=S2221-24 $342016000200010 \&$ Ing=es.

41. Lagos A. Un millón de hondureños padecen de diabetes. El Heraldo [Internet]. 7 de marzo de 2014 [Consultado 22 Noviembre 2017]. País.

Disponible en: http://www.elheraldo. hn/alfrente/566401-209/un-millon-de -hondurenos-pa decen-de-diabetes. 